\title{
Model-based fault detection and diagnosis optimization for process control rig
}

\begin{abstract}
One of the challenges research on model based fault detection and diagnosis of a system is finding the accurate models. In this paper, fuzzy logic based model using genetic algorithm for optimizing the membership function is used in the development of fault detection and diagnosis of a process control rig. The model is used to generate various residual signals, which relate to the faults of the system. These residual signals are used by artificial neural networks to classify the respective faults and finally to determine the faults of the system. Comparisons of the fault classification technique are done for two different models of the process control rig that are the conventional fuzzy model and the optimized fuzzy-GA model. The results show that the fuzzy-GA model gives more accurate fault classifications as compared to the conventional fuzzy logic model.
\end{abstract}

Keyword: Fault detection and diagnosis; Fuzzy logic; Genetic algorithms; Neural network; Process control 